\title{
RESULTADO DEL TRATAMIENTO HOMEOPÁTICO EN LA CALIDAD DE VIDA DEL PACIENTE PEDIÁTRICO CON DERMATITIS ATÓPICA
}

\author{
Adriana Ines Carolina Garcia Mesa \\ Médico y Cirujano Universidad Militar Nueva Granada. Candidata a título de \\ Magister Medicina Alternativa con énfasis en Homeopatía Universidad Nacional de Colombia \\ Correspondencia: mesaines@gmail.com
}

Recibido: Julio 2 de 2013 Aceptado: Noviembre 1 de 2013

\begin{abstract}
Resumen
La dermatitis atópica es una dermatosis pruriginosa crónica, cuya prevalencia se ha triplicado en los países industrializados durante las últimas tres décadas. La forma de manifestación y tiempo de evolución de esta patología se ha relacionado con un deterioro en la calidad de vida de los pacientes y su familia. Objetivos: Evaluar los efectos y resultados del tratamiento homeopático como manejo complementario o alternativo en la calidad de vida del paciente pediátrico con dermatitis atópica.

Métodos: Se realizó una búsqueda crítica de literatura en las siguientes bases de datos PUBMED, MEDLINE, EMBASE, SCIENCE DIRECT, SPRINGERLINK y OVID- JOURNAL, utilizando las palabras clave: Homeopatía, dermatitis atópica, calidad de vida, pacientes pediátricos.

Resultados: Revisión de estudios de cohorte multicéntrico prospectivo, estudio de observación clínica, y estudio observacional prospectivo de Europa y América latina sobre los efectos del tratamiento homeopático individualizado en la calidad de vida del paciente con dermatitis atópica.

Conclusiones: La homeopatía como tratamiento alternativo o complementario en el paciente pediátrico con dermatitis atópica, ha mostrado una mejoría significativa de los síntomas asociados a esta afección mórbida y su repercusión en el estado general del paciente tanto anímico, afectivo y social y por ende la mejoría en la calidad de vida de sus familiares también. En los estudios al comparar tratamiento convencional y homeopatía, no se encuentra diferencias significativas en los resultados, esto demuestra la eficacia del tratamiento homeopático, no efecto placebo, y sin efectos adversos.
\end{abstract}

Palabras clave: Homeopatía, calidad de vida, paciente pediátrico, dermatitis atópica, eccema.

\section{HOMEOPATHIC TREATMENT OUTCOME IN THE QUALITY OF LIFE OF PEDIATRIC PATIENTS WITH ATOPIC DERMATITIS}

\begin{abstract}
Atopic dermatitis is a chronic pruritic dermatosis whose prevalence has tripled in industrialized countries over the past three decades. The form of appearance and duration of this disease has been linked to deterioration in the quality of life of patients and their families.

Objectives: To evaluate the effects and results of homeopathic treatment as complementary or alternative management on the quality of life of pediatric patients with atopic dermatitis.

Methods: We performed a literature critical search in the following databases: PUBMED, MEDLINE, EMBASE, SCIENCE DIRECT, SPRINGERLINK and OVID-JOURNAL, by using the keywords: Homeopathy, atopic dermatitis, quality of life, pediatric patients.
\end{abstract}


Results: Review of prospective multicenter cohort studies, clinical observational study, and prospective observational study in Europe and Latin America over the effects of individualized homeopathic treatment on quality of life of patients with atopic dermatitis.

Conclusions: Homeopathy, as an alternative or complementary treatment in pediatric patients with atopic dermatitis, has shown a significant improvement in the symptoms associated with this disease and their effects on overall, mood, emotional and social conditions, and therefore enhancement in the quality of life for their families too. In studies comparing conventional treatment and homeopathy treatment there is no significant difference in the results; this demonstrates the efficacy of homeopathic treatment, with no placebo effect, and no adverse effects.

Keywords: Homeopathy, quality of life, pediatric patients, atopic dermatitis, eczema.

\title{
RESULTADO DO TRATAMENTO HOMEOPÁTICO NAQUALIDADE DE VIDA DO PACIENTE PEDIÁTRICO COM DERMATITE ATÓPICA
}

\begin{abstract}
Resumo
A dermatiteatópicaéuma dermatose pruriginosa crônica, cuja prevalênciatem-se triplicado nos países industrializados durante as últimas três décadas. A forma de manifestação etempo de evolução desta patologiatem-se relacionado com umdeterioro na qualidade de vida dos pacientes esuafamília.

Objetivos: Avaliar os efeitos e resultados do tratamento homeopático como manejo complementar ou alternativo na qualidade de vida do paciente pediátrico com dermatiteatópica.

Métodos: Se realizouumaprocura crítica de literatura nas seguintes bases de dados PUBMED, MEDLINE, EMBASE, SCIENCE DIRECT, SPRINGERLINK e OVID- JOURNAL, utilizando as palavras chaves: Homeopatia, dermatiteatópica, qualidade de vida, pacientes pediátricos.

Resultados: Revisão de estudos de cohortemulticêntrico prospectivo, estudo de observação clínica, eestudo observacional prospectivo da Europa eda América Latina sobre os efeitos do tratamento homeopático individualizado na qualidade de vida do paciente com dermatiteatópica.

Conclusões: A homeopatia como tratamento alternativo ou complementar no paciente pediátrico com dermatiteatópica, tem mostrado umamelhora significativa dos sintomas associados a esta afecção mórbida esua repercussão no estado general do paciente tanto anímico, afetivo e social e por tal motivo, amelhora na qualidade de vida de suasfamiliarestambém, nos estudos ao comparar tratamento convencional e homeopatia, não se encontramdiferenças significativas nos resultados, isto demonstra a eficácia do tratamento homeopático, não efeito placebo, esemefeitos adversos.
\end{abstract}

Palavras chave: Homeopatia; qualidade de vida; paciente pediátrico; dermatiteatópica; eccema.

\section{Introducción}

La dermatitis atópica (DA), es una patología que se caracteriza por una inflamación crónica de la piel, cuya etiopatogenia tiene múltiples factores involucrados, como: los cambios en el ambiente, la contaminación, los hábitos de higiene y la predisposición genética. Se considera como una de las enfermedades más prevalentes de la población pediátrica, debido al aumento de la incidencia durante las últimas tres décadas; entre un 15 a un $30 \%$ de los niños y de 2 a $10 \%$ de los adultos se encuentran afectados, por lo cual se han considerado como la epidemia no infecciosa del siglo XXI $(1,2,3,4,5,6,7,8,9)$.

La DA se caracteriza por lesiones en piel distribuidas en el cuerpo dependiendo de la edad, prurito intenso de predominio nocturno que puede alterar el ciclo de sueño-vigilia y por un curso dado por remisiones y exacerbaciones, que la hacen potencialmente debilitante 
y que compromete la calidad de vida del infante. Los síntomas, suelen aparecer en el $60 \%$ de los infantes en el primer año de vida, en el $85 \%$ a los 5 años, y un $10 \%$ inician la enfermedad después de los 7 años. La DA puede resolver en la adolescencia en el $50 \%$ de los pacientes, en el otro porcentaje persisten hasta la adultez $(1,2,3)$.

Estudios en psiquiatría mostraron que la DA es una de las alteraciones que mayor morbilidad psiquiátrica acumula, esto se explica debido a que los eventos estresantes son procesados e interpretados por cada individuo y de acuerdo con esa interpretación, se genera una respuesta que cumple una función adaptativa. De la respuesta, pueden surgir sentimientos negativos de miedo, cólera, rabia, depresión, indefensión y desesperanza, al igual que una depresión o supresión en la actividad del sistema inmunitario, secundario a mecanismos bioquímicos, a nivel del hipotálamo, hipófisis y glándulas suprarrenales. Si la respuesta adaptativa a estas situaciones se mantiene durante un periodo de tiempo prolongado, favorece la aparición de enfermedades, entre ellas la dermatitis atópica. La ansiedad y la depresión son algunos de los trastornos, más frecuentemente relacionados con la DA $(10,11,12$, $13,14,15,16,17,18)$.

Los pacientes con DA tienden a ser irritables, resentidos, hostiles, ansiosos, el niño suele ser demandante y con mayor necesidad de afecto. Son personas con problemas para expresar sus emociones, que internalizan sus problemas y expresan su ansiedad y hostilidad mediante el rascado de las lesiones, estas manifestaciones alteran la calidad de vida de este individuo, entendiéndose esta como un estado de satisfacción general, derivado de la realización de las potencialidades de la persona, que contempla una sensación subjetiva de bienestar físico, psicológico y social $(10,11,12,13,19,20,21,22)$.

El tratamiento convencional tiene en primera línea el uso de corticoides, cuyo efecto inmunosupresor, anti-inflamatorio, anti-proliferativo y vasoconstrictor, reducen el rascado y mejoran la apariencia de la piel, pero a largo plazo se puede presentar complicaciones como: la atrofia cutánea, las estrías, telangectasias, dermatitis de contacto, síndrome de Cushing, retardo del crecimiento, y supresión del eje hipotálamo-pituitaria-adrenal. Cuando los pacientes son refractarios a los corticoides tópicos, se inician tratamientos de segunda línea como los inmunomoduladores, antidepresivos, sedantes y fototerapia $(1,2,23,24,25,26)$.
La perturbación de la calidad de vida del paciente pediátrico con DA y de su familia, la preocupación por los efectos adversos de los corticoides y la insatisfacción con el tratamiento convencional, ha hecho de las medicinas y terapias alternativas y complementarias una opción de tratamiento. La Homeopatía es una de las medicinas alternativas más populares usadas en niños $(27,28,29,30,31)$.

La Homeopatía es un sistema médico complejo fundado por Samuel Hahnemann. Su principio filosófico es el Vitalismo, que entiende el concepto de salud-enfermedad a partir de la fuerza vital, la energía que anima el cuerpo y gobierna su funcionamiento, aquella que permite la unión del organismo al espíritu en una relación armónica y dinámica. El concepto de enfermedad para la Homeopatía es el desequilibrio o la perturbación de la energía vital, es la alteración de la armonía, que se expresa o manifiesta a través de los signos y síntomas que se relatan y que se puede observan en una persona $(32,33,34,35,36,37)$.

Su principio terapéutico y de curación es restablecer el equilibrio de la energía vital, siempre basándose en los fundamentos de la individualidad, la semejanza, la experimentación en personas sanas, el remedio único y la mínima dosis (34).

\section{Materiales y métodos}

Se realizó una búsqueda crítica de literatura en las siguientes bases de datos PUBMED, MEDLINE, EMBASE, SCIENCE DIRECT, SPRINGERLINK y OVIDJOURNAL, utilizando las palabras clave: Homeopatía, dermatitis atópica, eccema, calidad de vida, niños, pacientes pediátricos. En la búsqueda se hizo énfasis en la elección de trabajos en los cuales el tratamiento homeopático cumpliera con los principios de remedio único, individualidad y semejanza y que hicieran uso de escalas de medición de calidad de vida reconocida para patologías alérgicas.

\section{Resultados: Homeopatía, calidad de vida y dermatitis atópica.}

La búsqueda realizada en las diferentes bases de datos, dio como resultado el hallazgo de estudios que involucran diferentes metodologías con el fin de evaluar los efectos de la Homeopatía como medicina alternativa o complementaria en la calidad de vida de los pacientes pediátricos con dermatitis atópica. 
En Alemania y Suiza se desarrolló un estudio de cohorte multicéntrico prospectivo, cuyo objetivo era determinar el espectro de diagnósticos y tratamientos, así como el curso y evolución de aquellos pacientes que eligieron recibir el tratamiento homeopático. Los pacientes que participaron fueron seguidos por un total de 24 meses, las evaluaciones se realizaron mediante cuestionarios estandarizados: KITA, KINDL y MOS SF-36. Se diligenció el cuestionario antes de iniciar el tratamiento, y los controles se realizaron a los 3, 12 y 24 meses.

3.981 pacientes quienes cumplían con los criterios de inclusión decidieron participar en el estudio, 2851 eran adultos y 1130 niños. La mayoría de estos pacientes ya habían recibido tratamiento convencional previo. En los resultados del estudio se encontró que los diagnósticos más comunes fueron en la mujer la migraña, en el hombre la rinitis alérgica, y en los niños de ambos géneros la dermatitis atópica. La toma de los medicamentos convencionales disminuyó de $45 \%$ de la línea base (adultos 50,2\%, niños $14,2 \%$ ) a $26.8 \%$. (Adultos 31.8\%, niños 14.2\%), después de 24 meses. Según la valoración de los pacientes la severidad de la enfermedad disminuyó significativamente y de acuerdo con los terapeutas el 25,7\% de los diagnósticos no estuvieron presentes al completar los 2 años, como en la población pediátrica, que al finalizar los 2 años solo 574 pacientes continuaban bajo tratamiento. La calidad de vida en los infantes mostró una marcada mejoría durante el periodo de observación, en los escolares y adolescentes la mejoría en la calidad de vida soló fue visible durante los tres primeros meses de estudio. Tanto en las evaluaciones de pacientes y terapeutas, los pacientes jóvenes tuvieron una mejoría superior a los pacientes adultos y en las enfermedades severas se evidenció una mejoría mayor, que en las enfermedades menos severas (38).

En Japón, basándose en la premisa de los múltiples factores que contribuyen tanto en el inicio, evolución o como causa de las enfermedades crónicas en la piel. Los factores hereditarios, locales y psicológicos, a los cuales hacen énfasis como el estrés emocional, eventos estresantes de la vida y los hostigamientos diarios. Se realizó un estudio de observación clínica sobre los efectos del tratamiento homeopático individualizado en 60 pacientes entre los 14 y los 77 años, con enfermedades crónicas de la piel. Los participantes recibieron tratamiento homeopático individualizado, en conjunto al tratamiento dermatológico convencional en un periodo de 3 meses a 2 años y 7 meses, con control cada 3 meses. El 25\% de los pacientes tenían dermatitis atópica, el otro porcentaje tenía otras afecciones en piel (psoriasis, eccema no dermatitis atópica, acné y urticaria) y la gran mayoría había recibido tratamiento convencional, Medicina Tradicional China y psicoterapia, con resultados parciales. Al finalizar la consulta y tras realizada la repertorización, se prescribieron los remedios homeopáticos sobre la base de la individualidad, en potencias centesimal y milesimal. Puls, Sulph, Lyc, Sep, Nat-m, Ars, Calc-c, Merc, Mez, Bry, Caust, Phos, Staph, Alum, Apis, Carbo-v, Carc, Kali-c, Lach, Nat-c, Nux-v, Psor y Silica. A lo largo del estudio, los remedios fueron cambiados en varios pacientes, de acuerdo con la evolución y los requerimientos que se observaban en los controles (39).

La efectividad del tratamiento fue medido a través de la escala VAS (Escala visual análoga), tanto por los pacientes como por el médico tratante. La escala valora la evolución de las lesiones físicas y sus síntomas asociados, incluyendo la relación consigo mismo y el entorno. El $88 \%$ de los pacientes con DA y el $95 \%$ de los pacientes con los otros diagnósticos en piel mostraron una mejoría del $50 \%$ tanto en la impresión general como en su condición en piel, el 79,2 \% de los pacientes que se quejaban del prurito y alteración del sueño, reportaron una reducción del $50 \%$ de estos síntomas. Con respecto, a satisfacción en la vida diaria, el cumplimiento en el trabajo y la satisfacción en las relaciones humanas, cerca del $50 \%$ de los participantes reportaron mejoría. No se presentaron agravaciones homeopáticas severas (39).

Eizayaga y colaboradores, desarrollaron un estudio observacional prospectivo, con el objetivo de evaluar la efectividad del tratamiento homeopático en pacientes con dermatitis atópica (DA), entre junio del 2003 hasta febrero del 2006. La población: pacientes de la consulta externa de la Universidad de Maimonides y de la consulta privada de los terapeutas participantes en el estudio. Se hizo uso de la escala VAS (escala visual análoga), para la evaluación de los resultados. Los buenos respondientes se definieron como aquellos individuos que reportaban curación, estar "mucho mejor" o "mejor" o cambios en la escala de 30 o más milímetros de la línea base. Los homeópatas eran libres de elegir el tratamiento (remedio, dosis, potencia) y realizar los cambios necesarios a lo largo del estudio. Dentro de los remedios más frecuentemente prescritos se encuentra Sulph, Lyc, Sil, Merc, Calc, Graph, Morg-p, Ars, Psor, Puls, Nat-m, Phos. 42 pacientes entre los 2 
meses y los 66 años entraron al estudio, en la mayoría de los casos la intensidad era de moderada a severa, asociada a comorbilidades atópicas (asma y rinitis), y en tratamiento convencional simultaneo (40). Solo 26 de los pacientes regresaron a los controles, a 8 de los no asistentes se les realizo seguimiento telefónico.

Al final de los controles se evidenció una mejoría clínica y estadística tanto en las lesiones en piel, como en las escalas de calidad de vida. El 29,8\% de los pacientes manifestaron agravaciones homeopáticas transitorias, del tratamiento convencional solo el uso de los emolientes, tuvo una reducción estadística significativa. En el caso de los pacientes que no asistieron a control, pero con seguimiento telefónico, se encontraban curados o se sentían mejor (40).

Otro estudio de observación clínica se realizó en San Paulo, Brasil, evaluando la eficacia del tratamiento homeopático individualizado en pacientes con afecciones dermatológicas entre 2007 y 2008. En el estudio participaron adultos y niños de la consulta externa, de la Asociación Paulista de Homeopatía. Tras la consulta se realizó la prescripción de un remedio único e individualizado, en potencia centesimal y cincuentamilesimal. Asociado a otras recomendaciones terapéuticas como la nutrición, evitar alérgenos potenciales, actividad física y el uso de hidratantes tópicos. No había límites para el número de cambios de la medicación, ni restricciones en la dosis o dilución. El seguimiento se realizó durante 3 meses y fue realizado de manera personal por un integrante del grupo de investigación, la evaluación se realizó mediante la escala Dermartology Quality of Life Index (DQLI), en la consulta inicial, al mes, 3 y 6 meses de tratamiento.

De la población; 49 pacientes; el 35\% eran niños y $65 \%$ adultos. Los motivos de consulta fueron, dermatitis atópica $36,7 \%$, psoriasis $16 \%$, urticaria crónica $8 \%$, otros eccemas. El análisis final se realizó sobre 33 pacientes, ya que 15 de los participantes no asistieron a los seguimientos, por causa desconocidas o dificultad en adquirir la cita control y 3 personas descontinuaron la intervención por la agravación homeopática, a pesar de la explicación del terapeuta. El diligenciamiento del cuestionario fue insatisfactorio, algunos pacientes reportaban un empeoramiento en la calidad de vida en relación con su problema dermatológico, mientras en la consulta, se mostraba mejoría y expresaban satisfacción con la evolución. En los resultados se reporta una respuesta positiva en el 59\% de los casos posterior al tratamiento, no solo en la condición dermatológica, sino también en el estado general y una mejoría en el estado psicológico. 6 pacientes quienes no asistieron a los controles, por difícil acceso a las citas o por no consideran necesario el seguimiento, de manera telefónica reportaron mejoría. En el grupo con respuesta positiva, 5 suspendieron el uso de antihistamínicos y 4 descontinuaron el uso corticoide sistémico y tópico (41).

En Italia, mediante un estudio de cohorte observacional prospectivo, se propone indagar sobre las características sociodemográficas, los remedios homeopáticos de más frecuente prescripción y la evolución de los pacientes que habían recibido tratamiento homeopático en la Clínica Homeopática de Lucca, entre 1998 a 2008, evaluación realizada 5 a 8 años después de la primera consulta. De los pacientes valorados en ese periodo de tiempo en la clínica homeopática, 551 fueron pacientes pediátricos, de los cuales 213 tenían enfermedades atópicas, que incluían dermatitis (76), asma (83), rinitis alérgica (51) e intolerancia a los alimentos (3).

El protocolo de tratamiento en la clínica consistía, en la administración de un remedio único iniciando con potencia cincuentamilesimal (6Q). La evaluación o el seguimiento de los casos se realizaba a través de la escala Glasgow Homeopathic Hospital Outcome Score (GHHOS):

\section{$0=$ NINGUNO \\ $1=$ MEJORÍA LEVE \\ $2=$ MEJORÏA MODERADA \\ $3=$ MEJORÍA IMPORTANTE \\ $4=$ CURACIÓN/REGRESO A LO NORMAL y \\ $-1=$ EMPEORAMIENTO LEVE.}

De los 213 infantes, la mayoría menores de 14 años, el $80 \%$ habían utilizado tratamiento convencional y solo el 31\% había recibido tratamiento homeopático previo. Se obtuvo comunicación telefónica con tan solo 40 de los padres, de los niños con dermatitis atópica tratados con homeopatía, 28 de los 40 (70\%), tuvieron una completa remisión de la dermatitis atópica, mientras 12 de los 40 (30\%) aún continuaban con las lesiones en piel. De los pacientes que persistían con la dermatitis, su severidad era de leve a moderada, ninguna de intensidad severa. De los menores con enfermedades atópicas evaluados durante 1998 a 2000, 
los de mejor evolución fueron aquellos con dermatitis atópica, 73,5\% (GHHOS 3-4) (42).

En Austria se llevó a cabo un estudio observacional prospectivo multicéntrico, con el objetivo de evaluar la eficacia real del tratamiento homeopático clásico y su potencial para reducir la dosis del medicamento convencional. Los pacientes incluidos en el estudio tenían una edad mínima de 9 años, con diagnóstico de neurodermatitis, rinitis alérgica, conjuntivitis alérgica y asma bronquial. El paciente era evaluado mediante la escala VAS (escala visual análoga). El seguimiento se hizo en un periodo de 2 a 16 semanas (43). En total fueron reclutados 40 pacientes, el $17.5 \%$ de los pacientes estaban en edades entre los 9 a los 18 años. 90\% de los pacientes tenía rinitis alérgica, $60 \%$ conjuntivitis alérgica, $47,5 \%$ asma bronquial y $22.5 \%$ neurodermatitis. En la mitad de los pacientes la enfermedad había persistido por más de 10 años. Tras la consulta los pacientes recibieron su remedio constitucional, solo el $27.5 \%$ de los pacientes recibió además de su medicamento constitucional un remedio isopatico. El $61.9 \%$ de los pacientes descontinuaron al menos uno de los componentes del tratamiento convencional, $38 \%$ presentaron una reducción de la dosis y $9.5 \%$ tuvieron que incrementar la dosis de al menos un componente del tratamiento convencional. El 97, 5\% de los pacientes quisieron continuar con la terapia (43).

En Alemania, con el objetivo de evaluar la influencia del tratamiento homeopático tanto en los síntomas y signos del eccema, así como en la calidad de vida del paciente atópico en comparación con el tratamiento convencional, se realizó un estudio prospectivo multicéntrico de cohorte comparativo. El estudio se realizó entre 1998 y 2000, la población, jóvenes menores de 17 años, que no hubieran sido tratados previamente y que cumplieran con los criterios de inclusión: Prurito asociado al menos a 3 de 5 criterios: 1) eccema en pliegues, 2) piel seca por lo menos durante los últimos 12 meses, 3) historia de asma y rinitis alérgica, 4) antecedentes familiares de eccema (niños entre 1- 3 años de edad), 5) primer signo de enfermedad antes del primer año. Padres y pacientes eligieron el tratamiento de su elección (homeopatía o convencional). El tratamiento Homeopático incluía, la prescripción del remedio único basado en la ley de la semejanza. Las escalas de calidad de vida utilizadas para la valoración fueron Atopie Lebensqualitaets-Fragebogen (ALF), la escala KINDL y KITA. Los controles se llevaron a cabo a los 6 y 12 meses de iniciado el tratamiento. Al final se analizaron 118 niños con DA; 54 fueron tratados con homeopatía y 64 con manejo convencional.

Con respecto a la severidad de los síntomas en los dos grupos de tratamiento se evidenció una marcada mejoría de la condición del paciente. Padres y pacientes manifestaron una mejoría gradual de los síntomas en ambos grupos terapéuticos, al igual que lo manifestado por los terapéutas. En el grupo de Homeopatía se encontraron casos más graves. En cuanto a las escalas de calidad de vida no se encontró diferencia significativa de los resultados encontrados en los grupos terapéuticos (44).

Otro estudio observacional multicentrico comparativo, fue realizado para comparar la eficacia y costo del tratamiento homeopático clásico versus el tratamiento convencional en niños con dermatitis atópica, con controles a los 6 y 12 meses de iniciado el tratamiento. En los controles se evaluaba el prurito, las alteraciones del sueño, la calidad de vida y los costos. Fueron reclutados 135 niños entre el año y los 14 años, en el grupo terapéutico convencional 87 y con tratamiento homeopático 48. En el grupo de homeopatía se encontraban pacientes con casos más severos y con un tiempo mayor de evolución de la enfermedad. A los 6 y 12 meses de control no se encontró ninguna diferencia significativa entre los dos grupos, en cuanto a las manifestaciones y la calidad de vida. El tratamiento homeopático era más costoso (45).

\section{Discusión}

Basándose en los principios de la homeopatía, individualidad, semejanza, remedio único, la mayoría de las revisiones son estudios observacionales, puesto que para evaluar la eficacia del tratamiento homeopático y su influencia sobre la calidad de vida de los pacientes, no se debe generalizar una misma terapéutica, ni un mismo medicamento aunque la patología sea la misma, esto se vio demostrado en los estudios con el amplio espectro de remedios homeopáticos utilizados en los tratamientos y la selección de pequeñas muestras poblacionales debido a la metodología del tratamiento homeopático basada en la individualidad. Esto nos lleva a indicar la importancia de los controles presenciales en el tratamiento homeopático dentro de la metodología de los estudios, puesto que la comunicación entre el terapeuta y el paciente es de vital importancia, es la guía en el plan terapéutico hacia la curación, y se basa en el conocimiento que el paciente tenga de sí mismo 
y de la observación que haga de su sintomatología para informarlas al médico tratante y por supuesto del examen físico realizado durante la consulta.

Es por esto por lo que en la gran mayoría de las revisiones, se presenta cierta dificultad para evaluar la calidad de vida de los pacientes tratados con homeopatía, la calidad de vida en un paciente pediátrico con dermatitis atópica, incluye más aspectos, que la mejoría del prurito y de las lesiones en piel, conlleva también una sensación subjetiva de bienestar y armonía consigo mismo y su entorno. Y aunque existen cuestionarios estandarizados con esta finalidad, medir la eficacia del tratamiento homeopático y su influencia en la calidad de vida del paciente pediátrico con dermatitis atópi$\mathrm{ca}$, y que fueron utilizadas en la gran mayoría de las revisiones, valorando evolución de las lesiones físicas y sus síntomas asociados, la relación consigo mismo y el entorno, la impresión general, la mejoría de la condición en piel, la reducción del prurito, la reducción de las alteraciones del sueño, la satisfacción en la vida diaria, el cumplimiento en el trabajo, la satisfacción en las relaciones humanas y la discontinuidad en el tratamiento convencional. Es difícil generalizar o tratar de interpretar la calidad de vida, puesto que su definición es subjetiva al igual que el individuo mismo. Como lo mostraron los estudios de Eizayaga.y Waisse, Costa, sobre las inconsistencias que se presentaban entre lo diligenciado en los cuestionarios y lo expresado por el paciente durante la consulta presencial, o los pacientes que abandonan el estudio, o no siguen los controles, que en el análisis final se tildan como resultados negativos, al hacer contacto con estos individuos, la mayoría no regresa, porque están mejor o están curados.

Además, las afectaciones mórbidas en piel, como, la dermatitis atópica, están ligadas íntimamente al estado psicológico del individuo, por tanto, es difícil evaluar la eficacia de un tratamiento en una condición cuya causa o exacerbación se ve influenciada por tantos factores externos, que terminan siendo variables dentro del estudio que no se pueden aislar. Una de ellas, es la presencia asociada de comorbilidades alérgicas en los participantes de los diversos estudios como el asma y la rinitis. En Gründling, Schimetta. Real-life effect of classical homeopathy in the treatment of allergies: A multicenter prospective observational study, el $60 \%$ de los pacientes tenían manifestaciones asociadas como exantemas alérgicos no dermatitis y desordenes psicológicos, desorden de ansiedad y desorden disfórico en el $15 \%$ de los casos.
Por tanto, se debe promover la continuidad en los estudios de investigación sobre la Homeopatía, su efecto terapéutico, eficacia y eficiencia en los procesos alérgicos, por su alta prevalencia en la población, alta incidencia de morbilidad y deterioro de la calidad de vida de las personas que las desarrollan. Realizados por homeópatas de experiencia, con formulación individualizada, basada en una adecuada repertorización y teniendo en cuenta la totalidad sintomática, con muestras poblacionales de significancia estadística.

\section{Conclusiones}

El incremento en la prevalencia de las enfermedades alérgicas como la dermatitis atópica, tanto en países industrializados y en vías de desarrollo, su impacto en la calidad de vida de los pacientes y sus familias, la mejoría parcial con el tratamiento convencional y sus efectos adversos con uso prolongado, hace de la dermatitis atópica un proceso mórbido que requiere de un manejo complementario e integral, para recuperar la calidad de vida del individuo, por esto la medicina alternativa o completaría como la homeopatía, es una opción de tratamiento.

La eficacia de la Homeopatía en la dermatitis atópica radica en que su objetivo es estimular la vitalidad, aumentar la resistencia del propio organismo a la enfermedad, su capacidad de curarse y su respuesta inmune, no solo la mejoría de las lesiones en piel.

En los estudios se evidencia la selección de pequeñas muestras poblacionales debido a la metodología del tratamiento homeopático basada en la individualidad, por lo tanto, hace difícil la aplicación de los resultados a la población general. Sin embargo, en la gran mayoría de los estudios, y según la valoración de los pacientes, la severidad de la enfermedad disminuyó significativamente, y se mostró una mejoría tanto en la impresión general como en las lesiones en piel.

La homeopatía como tratamiento alternativo o complementario en el paciente pediátrico con dermatitis atópica, mostró una mejoría significativa de los síntomas asociados a esta afección mórbida y sus repercusiones en el estado general del paciente tanto, anímico, afectivo y social y por ende la mejoría en la calidad de vida de sus familiares también.

En los estudios al comparar tratamiento convencional y homeopatía, no se encuentra diferencias significativas en 


\section{RESULTADO DEL TRATAMIENTO HOMEOPÁTICO EN LA CALIDAD DE VIDA DEL PACIENTE PEDIÁTRICO CON DERMATITIS ATÓPICA}

los resultados, demostrando la eficacia del tratamiento homeopático, sin efecto placebo y sin efectos adversos.

\section{Agradecimientos}

Doctora Florencia Correa. Medico Homeópata. Magister en Medicina alternativa, Área Homeopatía, Universidad Nacional de Colombia. Docente Instituto de Homeopatía Luis G.Páez.

\section{Conflicto de intereses y financiación}

El artículo no tiene fuente de financiación.

\section{Referencias}

1. Gelbard C, Hebert A. New and emerging trends in the treatment of atopic dermatitis. Patient Preference and Adherence. 2008; 2: 387-392.

2. Ceruelo E. Dermatitis atópica: una enfermedad emergente. Rev Pediatr Aten Primaria. 2009; 11(15): 11-14.

3. Correale C, Walker C, Murphy L, Craig T. Atopic Dermatitis: A Review of Diagnosis and Treatment. Am Fam Physician. 1999; 60: 1191-210.

4. Zheng T, Yu J, Oh H, Zhu Z. The Atopic March: Progression from Atopic Dermatitis to Allergic Rhinitis and Asthma. Allergy Asthma Immunol Res. 2011; 3(2): 67-73.

5. Fierro A, Castañeda D. Marcha alérgico: el camino de la atopia. Alergia, Asma e Inmunologia Pediatricas. 2006; 15: 50-56.

6. Honeyman J. Dermatitis atópica. Atopia cutánea. (monografía en internet). Chile: Dermatologia Ibero-Americana Online; 2011 [acceso diciembre de 2012]. Disponible en: http://piel-l.org/ libreria/item $/ 1315$

7. Baena C, Lockey R. Inmunoterapia en América Latina. Del pasado al futuro. Revista Alergia México. 2008; 55(1): 33-37.

8. Martínez C. Ponencia: causas del aumento de las enfermedades alérgicas. Alergol Inmunol Clin. 2002; (17): 1-29.

9. Gomez M, Aldana E. Alteraciones psicológicas en la mujer embarazada de alto riesgo. Psicología y salud [revista en Internet]. 2007; 17: 53-61. [acceso diciembre de 2012]. Disponible en: http://redalyc.uaemex.mx/redalyc/pdf/291/29117106.pdf

10. Bernardo S, Garcia E, Gonzalez A. Aspectos psicológicos de los enfermos con dermatitis atópica: una revisión Clínica y Salud. Colegio Oficial de Psicólogos. 2002; 13: 285-306.

11. Chamlin S, Chren M. Quality-of-life outcomes and measurement in childhood atopic dermatitis. Immunol Allergy Clin North Am. 2010; 30(3): 281-288.

12. Shobaili $\mathrm{H}$. The impact of childhood atopic dermatitis on the patients' family. Pediatric Dermatology. 2010; 27(6): 618-623.

13. McKenna P, Doward L. Quality of life of children with atopic dermatitis and their families. Current Opinion in Allergy and Clinical Immunology. 2008; 8: 228-231.

14. Gómez B, Escobar A. La psiconeuroinmunología: bases de la relación entre los sistemas nervioso, endocrino e inmune. Rev Fac Med UNAM. 2002; 45: 22-27.
15. Montero J, Mullol J, Jáuregui I, Dávila I, Ferrer M, Bartra M. et al. Stress and allergy. Investig Allergol Clin Immunol. 2009; (19) 1: 40-47.

16. Hewitt N, Salamanca A. Factores biológicos y psicológicos de la dermatitis atópica. Psychologia: avances de la disciplina. 2011; (5) 1: 47-58.

17. Mohammad J. Psychodermatology: A guide to understanding common Psychocutaneous disorders. Prim Care Companion JclinPsychiatry. 2007; 9: 203-213.

18. Fontes P, Weber M, Forte S, Cestari T. Atopic dermatitis in infants: a psychosomatic approach. Rev. psiquiatr. Rio Gd. Sul [revista en Internet]. 2006; 28: 78-82. [acceso diciembre de 2012]. Disponible en: http://www.scielo.br/scielo.php?pi$\mathrm{d}=$ S01011082006000100010\&script $=$ sci_abstract

19. Ardila R. Calidad de vida: una definición integradora. Revista Latinoamericana de Psicología. 2003; 35: 161-164.

20. Urzúa A. Calidad de vida relacionada con la salud: Elementos conceptuales. Rev Med Chile. 2010; 138: 358-365.

21. Martínez A, Serrano P, López M, Contreras F. Calidad de vida en pacientes alérgicos. Alergol Inmunol Clin. 2000; 15: 184-203.

22. Izco M. Calidad de vida en pacientes alérgicos. Rev. Esp. Alergol Inmunol Clín. 1998; 13(2): 128-130.

23. Moreno J. Dermatitis atópica Alergol Inmunol Clin. 2000; 15 : 279-295.

24. Möhrenschlager M, Ring J. Atopic Eczema. Current Allergy and Asthma Reports. 2006, 6: 445-447.

25. Chang C, Keen C, Gershwin E. Treatment of Eczema. Clinic Rev Allerg Immunol. 2007; 33: 204-225.

26. Akdis C, Akdis M, Bieber T, Bindslev-Jensen C, Boguniewicz M, Eigenmann $P$, et al. Diagnosis and treatment of atopic dermatitis in children and adults: European Academy of Allergology and Clinical Immunology/American Academy of Allergy, Asthma and Immunology/PRACTALL. Consensus Report, Allergy. 2006: 61: 969-987.

27. Belory L. Complementary and Alternative Medicine population based studies: a growing focus on allergy and asthma. Allergy. 2002; 57: 655-658.

28. Spigelblat L. Homeopathy in the pediatric population. Pediatr Child Health. 2005; 10(3).

29. Ernst $E$. The usage of complementary therapies by dermatological patients: a systematic review. British Journal of Dermatology. 2000; 142: 857-861.

30. Birdee G, Phillips R, Davis R, Gardiner P. Factors associated with pediatric use of complementary and alternative medicine in the United States: Results from the national health interview survey. Pediatrics. 2010: 125(2): 249-256.

31. Hughes R, Ward D, Tobin A, Keegan K, Kirby B. The use of alternative medicine in pediatric patients with atopic dermatitis. Pediatric Dermatology. 2007; 24 (2): 118-120.

32. Alarcón A, Vidal A, Neira J. Salud intercultural: elementos para la construcción de sus bases conceptuales. Rev Méd Chile. 2003; 131: 1061-5.

33. Mendoza D. Racionalidades médicas. En: Diana Zulima Urrego Mendoz JAVO, editor. La medicina alternativa: una visión desde los sistemas médicos complejos Grupo de investigación racionalidades médicas y prácticas en salud enfermedad Maestría en medicina alternativa. 1 ed. Bogotá: Universidad Nacional de Colombia; 2011. p. 17- 27. 
34. Diaz J. Energía Vital y Farmacodinámica. En: Fundación Instituto Luis G.Paez, editor. Doctrina Homeopática. 1 ed. Bogotá: Fundación Instituto Colombiano de Homeopatía Luis G.Paez; 2005. p. 61-8.

35. Hahnemann S. El órganon de la medicina VI edición. México: Instituto Nacional de México. 2 edición; 2001.

36. Gupta R, Manchanda R. Libro de texto de Dermatología para Homeópatas. India: B. Jain Publishers; 2010.

37. Avello M, Avendaño C, Mennickent S. Aspectos generales de la homeopatía. Rev Méd Chile. 2009; 137: 115-120.

38. Witt. C, Lüdtke. R, Baur R, Willich S. Homeopathic medical practice: Long-term results of a cohort study with 3981 patients. BMC Public Healt; 2005; 5:115. [acceso diciembre de 1012]. Disponible en : http://www.biomedcentral.com/1471-2458/5/115

39. Itamura R. Effect of homeopathic treatment of 60 Japanese patients with chronic skin disease. Complementary Therapies in Medicine. 2007; 15: 115-120.

40. Eizayaga E, Eizayaga J. Prospective observational study of 42 patients with atopic dermatitis treated with homeopathic medicines. Homeopathy. 2012; 101: 21-27.
41. Waisse S. Jurj G. Costa L. Simone T. Labonia W. Sos A. et al. Individualized homeopathic treatment of dermatological complaints in a public outpatient clinic. Homeopathy. 2009; 98: 149-153.

42. Rossi E, Bartoli P, Bianch A, Da Fre M. Homeopathy in paediatric atopic diseases: long-term results in children with atopic dermatitis. Homeopathy. 2012; 101: 13-20.

43. Gründling C, Schimetta W, Frass M. Real-life effect of classical homeopathy in the treatment of allergies: A multicenter prospective observational study. Wien Klin Wochenschr. 2012; 124: 11-17.

44. Keil T, Witta C, Roll S, Vancea W, Webera K, Wegscheiderb K, et al. Homoeopathic versus conventional treatment of children with eczema: A comparative cohort study. Complementary Therapies in Medicine. 2008; 16: 15-21.

45. Witt B, Brinkhaus T, Reinhold S, Roll K, Wegscheider K, Wruck $\mathrm{D}$, et al. A comparative observational study to assess the effectiveness and costs of individualized homeopathy compared to conventional treatment in children with atopic dermatitis-An example for whole system research. En: Presentaciones orales. European Journal of Integrative Medicine. 2008; 1: 22. 